\title{
Unidad didáctica sobre los cambios químicos que intervienen en el efecto invernadero
}

\author{
Marina Martínez-Carmona y Luisa López-Banet \\ Universidad de Murcia. Dpto. de Didáctica de las Ciencias Experimentales
}

[Recibido el 18 de abril de 2021, aceptado el 27 de julio de 2021]

En este trabajo se proponen actividades secuenciadas como parte de una unidad didáctica sobre la enseñanza de los cambios químicos que intervienen en el efecto invernadero para abordar el aprendizaje de la Química con sentido y desarrollar, no solo la comprensión de los contenidos científicos, sino también cómo se construyen. En concreto, se ha diseñado con la intención de promover objetivos competenciales relacionados con los fundamentos de la disciplina que permitan dar respuesta a las cuestiones estructurantes surgidas del contexto implicado. La unidad didáctica integra actividades centradas en el alumnado, como investigaciones en las que se recogen e interpretan datos para el establecimiento de conclusiones, tareas de argumentación o el aprendizaje basado en juegos. Una prueba inicial con alumnado de 20 de ESO ha permitido establecer su idoneidad para este nivel educativo, resultando un recurso relevante y motivador para la introducción de los contenidos seleccionados.

Palabras clave: Educación Secundaria Obligatoria; innovación; argumentación; juego; perspectiva de género.

\section{Didactic unit on the chemical changes involved in the greenhouse effect}

This article reports on a didactic unit of sequenced activities on the chemical changes involved in the greenhouse effect. The purpose of the unit is to make chemistry learning meaningful for students and develop not only their understanding of the scientific content itself but also how the information is obtained. The activities are designed to equip students with competencies related to the fundamental principles of chemistry which will enable them to respond to structural questions arising from the context of study. The activities in the unit are student-focused and include research (data collection and interpretation and formulation of conclusions), argumentation and gamebased learning. An initial trial with second-year students showed the activities to be a suitable, relevant and motivating resource for introducing content of this type at this level.

Keywords: Compulsory Secondary Education; innovation; argumentation; game; gender perspective.

Para citar el artículo. Martínez-Carmona, M. y López-Banet, L. (2021). Unidad didáctica sobre los cambios químicos que intervienen en el efecto invernadero. Ápice. Revista de Educación Científica, 5(2), 71-85. DOI: https://doi.org/10.17979/arec.2021.5.2.7997

Contacto. marina.m.c1@um.es y llopezbanet@um.es 


\section{Introducción}

Las orientaciones curriculares en las que se basa el profesorado para la planificación docente suelen presentar los contenidos científicos de forma desconectada de las prácticas de investigación y de las habilidades tecnológicas, así como de las perspectivas personales y sociales, lo que promueve una enseñanza de la Química alejada de los fundamentos que la constituyen (Talanquer, 2013). La puesta en práctica de un currículum que aprovecha los problemas o situaciones del mundo real como enfoque integral que va más allá de las materias científicas, y el objetivo de brindar oportunidades al alumnado para asumir roles activos en el proceso de aprendizaje al abordar experiencias que llamen la atención sobre la ciencia, permite mejorar las actitudes del alumnado (Zhang y Campbell, 2012). Asimismo, el diseño de unidades didácticas competenciales requiere de un contexto de relevancia para el alumnado, que implique un conocimiento científico central en el que los contenidos y su secuenciación sean rigurosamente seleccionados (Couso, 2013). Esta propuesta para 20-3을 curso de Educación Secundaria pretende servir para la práctica docente de contenidos relacionados con los cambios químicos que intervienen en el efecto invernadero, teniendo en cuenta el modelo de aprendizaje competencial.

\section{Fundamentación teórica y revisión bibliográfica}

Las reacciones químicas son uno de los contenidos fundamentales en la enseñanza de la Química y tienen un valor relevante en el currículo de secundaria ya que permiten entender muchos de los fenómenos de la vida cotidiana (Aragón, Oliva y Navarrete, 2013). Los cambios químicos, además de explicar situaciones del mundo que nos rodea, permiten crear nuevas sustancias y diseñar procesos a través de los cuales materiales conocidos se transforman en productos de gran utilidad (Talanquer, 2013). El diseño de una propuesta para abordar estos contenidos implica que los docentes consideren estrategias de enseñanza específicas en línea con los objetivos de aprendizaje teniendo en cuenta las dificultades de aprendizaje que pueden implicar (Bayram-Jacobs et al., 2019). En Química, el alumnado sigue su sentido común y tiende a generar explicaciones rápidas de fenómenos naturales basadas en su intuición, realizando amplias generalizaciones, sin mucha reflexión (Talanquer, 2006). Otras dificultades pueden ser debidas a características intrínsecas y terminológicas de la propia disciplina, al pensamiento y procesos de razonamiento del alumnado o al proceso de instrucción recibido. Respecto a la disciplina, los tres niveles de descripción de la materia (observacional, microscópico y representacional) podrían interferir en el aprendizaje del cambio químico (Furió y Furió, 2000). En cuanto a las atribuibles al proceso de instrucción, destacaremos el uso de criterios de secuenciación inadecuados, así como la presentación de forma acabada de los conceptos y teorías (Caamaño y Oñorbe, 2004). En cuanto al razonamiento del alumnado respecto a los contenidos seleccionados, es destacable, por ejemplo, que un gran número de alumnos y alumnas, al hablar de lo que ocurre cuando un coche consume gasolina, conciben el cambio químico como la desaparición de productos, pero muy pocos hacen referencia a la aparición de los gases de escape (Andersson, 1990). Es comprensible que el alumnado difícilmente acepte la existencia de los gases en situaciones estáticas o en las que no observa alguna de sus propiedades, siendo considerados como algo con muy poco estatus material y que no se perciben, pero que sí lo haga cuando detecte alguna de sus acciones o efectos (Domínguez et al., 2000).

Respecto a cómo enseñar, algunos enfoques relevantes para la enseñanza de esta disciplina proponen que se introduzcan los conceptos de química fundamental según sean necesarios para comprender los problemas sociales, económicos o ambientales relevantes, como el calentamiento global o las fuentes de energía alternativas. De hecho, actual- 
mente se recomienda que, en lugar de insistir en explicar fenómenos, se debe centrar la atención en la enseñanza de cómo piensan los/as químicos/as y cuáles son sus modos de razonamiento, para poder comprender cómo esta rama de la ciencia ha permitido a la humanidad interactuar con más éxito con su entorno, incluyendo la discusión de las implicaciones socio-éticas de la manipulación química y la resolución de problemas reales (Talanquer, 2013). La competencia científica en el aula podría ser trabajada a partir de contextos relevantes en la vida diaria, que se encuentren en el entorno cultural del alumnado, capten su interés y permitan desarrollar un tratamiento didáctico en el aula (Franco-Mariscal, Blanco-López y España-Ramos, 2014; Blanco, España y Rodríguez, 2012; Caamaño, 2018). La selección de contextos adecuados implica que sean conocidos y relevantes para el alumnado, que no sean complicados, que no desvíen la atención de los conceptos relacionados y que no confundan. Por otro lado, tras la introducción del contexto, se debe realzar la relación del mismo con los conceptos relacionados, por ejemplo, por medio de actividades de indagación que promuevan la necesidad de saber, de encontrar respuestas a preguntas sobre conceptos relevantes, de relacionarlos con información de otras fuentes y de aplicar el conocimiento en sucesivos contextos (Jong, 2008).

Asimismo, el énfasis por abrir espacios para que el alumnado participe productivamente en las prácticas científicas ha impulsado a más docentes a involucrar al alumnado en actividades basadas en la indagación, modelización y argumentación (Domènech-Casal, 2017; Garrido y Couso, 2013; Jiménez-Aleixandre, 2010; Crujeiras, et al., 2013; Crujeiras y Jiménez-Aleixandre, 2018; Jiménez-Liso et al., 2018), reclamando la necesidad de generar actitudes positivas hacia la ciencia, desarrollar el pensamiento crítico y dudar de cualquier afirmación que no se sustente mediante pruebas (Jiménez-Liso, 2020). En definitiva, a diferencia de exponer el conocimiento disciplinario como ocurre en enfoques tradicionales, el planteamiento de un problema auténtico de la vida real que permita contextualizar el aprendizaje de la Química promueve el desarrollo de habilidades lógicas, críticas y creativas en el alumnado, así como la motivación, al dotar de sentido y utilidad a los contenidos que se van a estudiar (Bellová et al., 2018). Además, la Química goza de una buena posición en el tratamiento de los sesgos con un modelo de buenas prácticas, que desarrolle habilidades prácticas y promueva la formación en competencia cultural y de enfoques orientados a mejorar la igualdad, la diversidad y la inclusión (Mehta et al., 2018). Sin embargo, en muchas ocasiones se presupone que el alumnado ya tiene adquiridos los conocimientos científicos necesarios para abordar las prácticas científicas y el contexto es relegado a la fase final de aplicación de ideas. Por tanto, el objetivo de aprendizaje debe implicar que el alumnado sea capaz de construir modelos teóricos que sean útiles en otras situaciones (Marchán-Carvajal y Sanmartí, 2015).

Por otro lado, el uso de juegos didácticos es otra estrategia que produce avances importantes en el aprendizaje de contenidos, que es valorada positivamente por el alumnado y que puede contribuir a desarrollar actitudes positivas hacia el aprendizaje (Franco y Oliva, 2013). En los últimos años está aumentando el número de publicaciones que proponen el uso de este tipo de metodologías centradas en el alumnado y que permiten desarrollar y ejercitar una gran variedad de competencias (Gee, 2003), como las relacionadas con el pensamiento creativo, analítico y reflexivo, con aspectos prácticos del aprendizaje, con el autoconocimiento y con el trabajo colaborativo, habilidades sociales y comunicativas (Boghian et al, 2019).

A pesar de todas las propuestas mencionadas, los referentes sobre los contenidos básicos y el enfoque de la enseñanza de la Química como ciencia fundamental han permanecido prácticamente intactos (Talanquer, 2013). Aún existen pocos materiales educativos adaptados a impulsar la enseñanza del conocimiento de la naturaleza de la investigación 
científica (Strippel y Sommer, 2015), pues el profesorado de Química no suele incluir prácticas científicas en su enseñanza (Boesdorfer y Livermore, 2018). Con esta finalidad, el diseño de materiales curriculares puede resultar de utilidad para facilitar al profesorado la adaptación e implementación de nuevos enfoques en la práctica educativa, alternativa a la tradicional. Desde hace unos años, se vienen recomendando actividades diferenciadas y secuenciadas que partan de situaciones concretas y simples para iniciar un proceso de construcción de modelos en base a situaciones cada vez más abstractas y complejas por medio del establecimiento de cuatro momentos clave (Jorba y Sanmartí, 1994; Pujol, 2003). En cuanto al diseño de actividades de enseñanza para unidades competenciales, Couso (2013) propone que se aborden en base a las siguientes preguntas:

- “¿Qué queremos que los alumnos aprendan, teniendo en cuenta para qué queremos que lo aprendan?"

- “¿Qué les haremos pensar, comunicar, hacer y sentir/ser para que lo aprendan?"

Para dar respuesta a la primera pregunta resulta muy útil identificar el problema y, a partir de él, interrelacionar las nociones estructurantes del tema (Sanmartí, 2000). Concretamente, incluyendo cuestiones del tipo a las que guían el trabajo de la mayoría de los científicos y científicas y organizan los temas en un currículum más auténtico: ¿Qué es esto? (Preguntas de análisis), ¿Cómo lo hago? (Cuestiones de síntesis), ¿Cómo lo cambio? (Cuestiones de transformación) y ¿Cómo te lo explico? (Cuestiones de modelización) (Talanquer y Pollard, 2010). La respuesta a esta pregunta conduce a la elección de los contenidos, en este caso, los relacionados con el modelo de cambio químico en un contexto de interés cultural como es el efecto invernadero. Además, la secuenciación de los contenidos se puede abordar mediante la introducción de contextos relacionados que permitan la construcción del modelo teórico sobre el cambio químico (Marchán-Carvajal y Sanmartí, 2015).

La segunda pregunta se responde mediante la planificación de situaciones de aprendizaje que persigan un camino que facilite la construcción del modelo anterior, teniendo presente de dónde parte el alumnado, los hitos que debemos alcanzar, hasta dónde queremos llegar y la competencia científica global (Couso, 2013). Para ello, prestamos especial interés a grandes ideas (problemas sociales y ambientales) y a que experimenten la sensación de pensar y trabajar como los/as científicos/as, motivando una actitud crítica e interés por la ciencia.

\section{Diseño de la propuesta}

Teniendo en cuenta los referentes mencionados, presentamos una propuesta educativa dirigida al alumnado de segundo y tercer curso de la ESO de la asignatura Física y Química. La secuencia pretende armonizar el enfoque en contexto con orientaciones centradas en el alumnado, con la incorporación de la indagación, la modelización y la argumentación, impulsando la reflexión sobre la naturaleza de la química (Caamaño, 2018). En definitiva, la intención de esta propuesta es presentar una unidad didáctica competencial inspirada en el modelo de Couso (2013) para abordar el cambio químico con sentido para el alumnado mediante la contextualización, a través de situaciones de la vida cotidiana que permitan reconocer la utilidad del nuevo aprendizaje y expresar las ideas sobre el mismo (Marchán-Carvajal y Sanmartí, 2015). Además, la contextualización de los contenidos permite progresar de manera gradual en los componentes de la argumentación científica en el aprendizaje de las reacciones químicas, así como incorporar la alfabetización científica al aula (Ibacache y Merino, 2021).

Con la secuencia de actividades propuesta se pretende que el alumnado compruebe cómo cambian las propiedades de la materia cuando tiene lugar un cambio químico (como la 
temperatura de una mezcla de gases), que sea capaz de imaginar qué ocurre durante la reacción química y justificar a qué se deben esas diferencias, y que compruebe el efecto de la lluvia ácida por medio del diseño experimental y la toma de datos. En la figura 1 se recogen las nociones estructurantes que se abordan a lo largo de las distintas actividades incluidas en la propuesta.

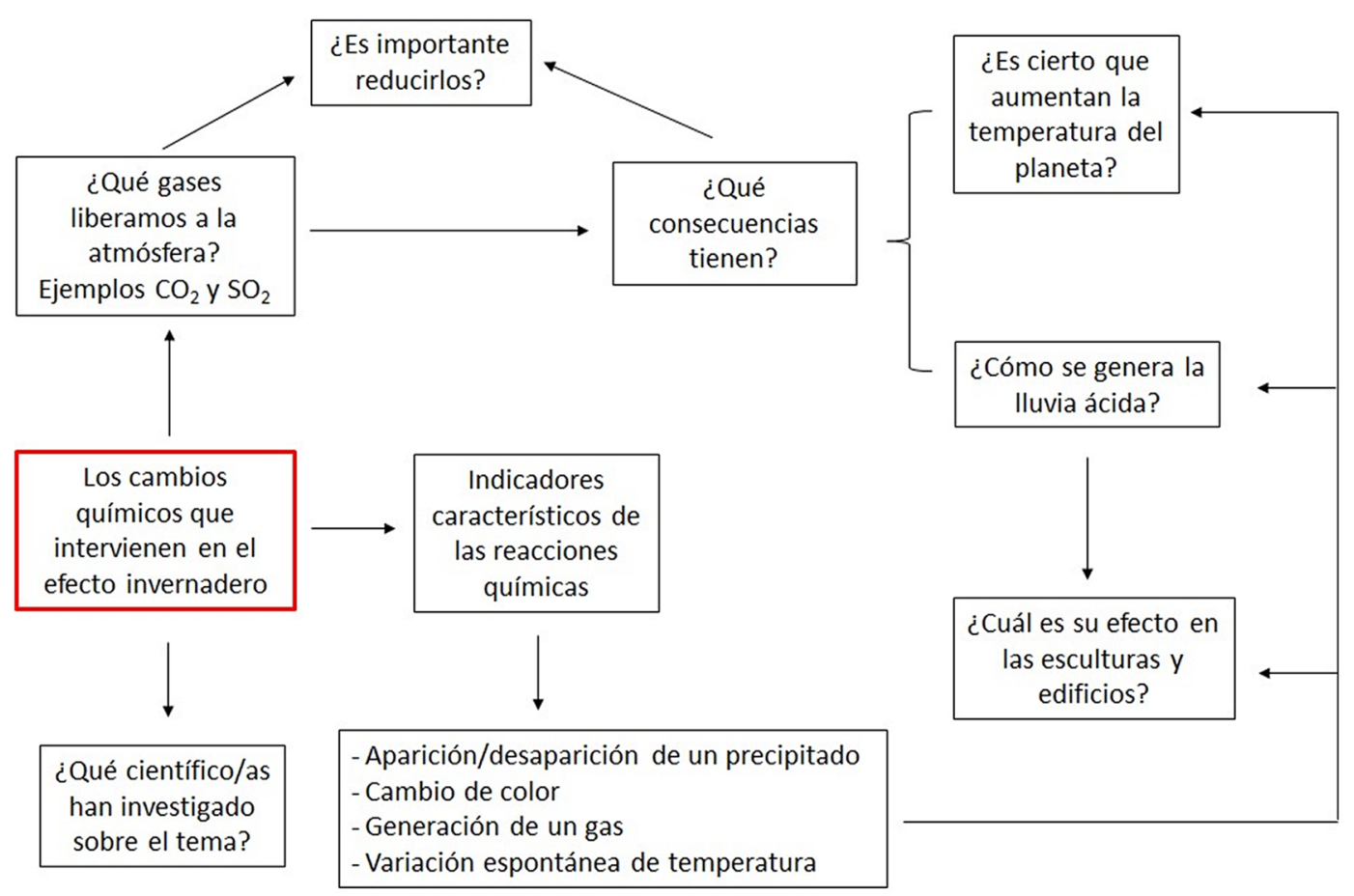

Figura 1. Nociones estructurantes del tema

Estos contenidos se concretan en la tabla 1 de acuerdo con los referentes recogidos por el Ministerio de Educación, Cultura y Deporte (2015) para los cursos 2ㅇ y 30 de Educación Secundaria Obligatoria de las materias de Física y Química, Matemáticas y Tecnología.

Las actividades que pretenden abordar los contenidos presentes en la tabla 1 se describen de forma más detallada en el anexo, en el que se muestran algunas imágenes de la puesta en práctica de la versión inicial de la propuesta con un grupo de estudiantes de 20 ESO. Esta posibilidad permitió que se introdujeran ciertas mejoras que constituyen la unidad didáctica que se presenta. 
Tabla 1. Actividades propuestas, contenidos, objetivos a desarrollar y recursos empleados

\begin{tabular}{|c|c|c|c|}
\hline Actividad & Contenidos & Objetivos & Recursos didácticos \\
\hline $\begin{array}{l}\text { 1: ¿Es cierto que } \\
\text { España se convertirá } \\
\text { en un gran desierto? } \\
\text { Científicos/as que } \\
\text { investigan sobre el } \\
\text { efecto invernadero } \\
\text { y sus consecuencias } \\
\text { relacionadas con el } \\
\text { cambio climático }\end{array}$ & $\begin{array}{l}\text { Científicos/as } \\
\text { relevantes. } \\
\text { Explicitación de la } \\
\text { perspectiva de género }\end{array}$ & $\begin{array}{l}\text { Contextualizar la } \\
\text { unidad didáctica } \\
\text { mediante un tema } \\
\text { de repercusión } \\
\text { económica, científica } \\
\text { y ambiental. } \\
\text { Concienciación sobre } \\
\text { la importancia del } \\
\text { papel que distintos } \\
\text { científicos y científicas } \\
\text { han jugado en } \\
\text { el avance de los } \\
\text { conocimientos sobre } \\
\text { el efecto invernadero } \\
\text { y sus consecuencias. } \\
\text { Visualización del } \\
\text { papel de la mujer en } \\
\text { la ciencia. } \\
\text { Adquisición } \\
\text { de habilidades } \\
\text { comunicativas. }\end{array}$ & Actividad interactiva \\
\hline $\begin{array}{l}\text { 2: ¿Cómo podríamos } \\
\text { averiguar si los } \\
\text { gases que expulsan } \\
\text { los vehículos hacen } \\
\text { que aumente la } \\
\text { temperatura por } \\
\text { efecto invernadero? }\end{array}$ & $\begin{array}{l}\text { Efecto invernadero } \\
\text { Identificación } \\
\text { de factores } \\
\text { característicos de una } \\
\text { reacción química: } \\
\text { generación de un gas } \\
\text { y modificación de la } \\
\text { temperatura. } \\
\text { Manejo de } \\
\text { dispositivos } \\
\text { (termómetro) } \\
\text { Elaboración de } \\
\text { hipótesis } \\
\text { Diseño experimental } \\
\text { Realización e } \\
\text { interpretación de } \\
\text { gráficas } \\
\text { Análisis de datos } \\
\text { Establecimiento de } \\
\text { conclusiones }\end{array}$ & $\begin{array}{l}\text { Desarrollar la } \\
\text { competencia } \\
\text { científica mediante } \\
\text { la elaboración de } \\
\text { hipótesis, diseño } \\
\text { experimental, toma } \\
\text { y análisis de datos } \\
\text { y elaboración de } \\
\text { conclusiones respecto } \\
\text { al efecto que tiene el } \\
\mathrm{CO}_{2} \text { en el aumento } \\
\text { de la temperatura del } \\
\text { planeta. } \\
\text { Generar un conflicto } \\
\text { cognitivo la variación } \\
\text { de temperatura. } \\
\text { Reflexión crítica sobre } \\
\text { las consecuencias. } \\
\text { Construcción } \\
\text { de aprendizajes } \\
\text { significativos }\end{array}$ & $\begin{array}{l}\text { Propuesta de } \\
\text { actividad de } \\
\text { indagación y } \\
\text { modelización }\end{array}$ \\
\hline
\end{tabular}


Tabla 1. Continuación. Actividades propuestas, contenidos, objetivos a desarrollar y recursos empleados

\begin{tabular}{|c|c|c|c|}
\hline Actividad & Contenidos & Objetivos & Recursos didácticos \\
\hline $\begin{array}{l}\text { 3: Generación de la } \\
\text { Iluvia ácida }\end{array}$ & $\begin{array}{l}\text { Lluvia ácida } \\
\text { Identificación } \\
\text { de factores } \\
\text { característicos de una } \\
\text { reacción química: } \\
\text { cambio de color y pH } \\
\text { de una disolución } \\
\text { Manejo de material } \\
\text { de laboratorio } \\
\text { Elaboración de un } \\
\text { informe }\end{array}$ & $\begin{array}{l}\text { Familiarización con } \\
\text { la metodología de } \\
\text { trabajo científica } \\
\text { incluyendo: } \\
\text { observación y } \\
\text { descripción de } \\
\text { fenómenos }\end{array}$ & $\begin{array}{l}\text { Trabajo práctico de } \\
\text { laboratorio }\end{array}$ \\
\hline $\begin{array}{l}\text { 4: ¿Duran menos los } \\
\text { monumentos por } \\
\text { causa de la lluvia } \\
\text { ácida? }\end{array}$ & $\begin{array}{l}\text { Efectos de la lluvia } \\
\text { ácida } \\
\text { Identificación } \\
\text { de factores } \\
\text { característicos de una } \\
\text { reacción química: } \\
\text { disolución de un } \\
\text { sólido } \\
\text { Elaboración de } \\
\text { hipótesis } \\
\text { Diseño experimental } \\
\text { Realización en } \\
\text { interpretación de } \\
\text { gráficas } \\
\text { Análisis de datos } \\
\text { Establecimiento de } \\
\text { conclusiones }\end{array}$ & $\begin{array}{l}\text { Aplicar los } \\
\text { conocimientos } \\
\text { adquiridos en un } \\
\text { contexto diferente } \\
\text { relacionado con los } \\
\text { contenidos de la } \\
\text { unidad didáctica. }\end{array}$ & $\begin{array}{l}\text { Propuesta de } \\
\text { actividad de } \\
\text { indagación y } \\
\text { modelización }\end{array}$ \\
\hline 5: Chem's up & $\begin{array}{l}\text { Todos los contenidos } \\
\text { anteriores }\end{array}$ & $\begin{array}{l}\text { Revisar y destacar los } \\
\text { principales contenidos } \\
\text { abordados en la } \\
\text { unidad didáctica. }\end{array}$ & Juego de cartas \\
\hline $\begin{array}{l}\text { 6: ¿Tienen razón los } \\
\text { negacionistas? }\end{array}$ & $\begin{array}{l}\text { Actitud crítica } \\
\text { Argumentación }\end{array}$ & $\begin{array}{l}\text { Justificar críticamente } \\
\text { los argumentos a } \\
\text { favor o en contra } \\
\text { del cambio climático } \\
\text { y aumento de la } \\
\text { temperatura por } \\
\text { efecto invernadero. }\end{array}$ & $\begin{array}{l}\text { Lectura de } \\
\text { información sobre } \\
\text { producción de } \\
\text { energía mediante } \\
\text { hidrocarburos y } \\
\text { planteamiento de } \\
\text { preguntas }\end{array}$ \\
\hline
\end{tabular}




\section{Consideraciones finales y limitaciones de la propuesta}

En consonancia con Franco-Mariscal, Blanco-López y España-Ramos (2014), consideramos que es necesario proponer materiales didácticos para que el profesorado disponga de ejemplos fundamentados con los que contribuir al desarrollo de la competencia científica en el alumnado al tiempo que fomenta su interés por la Química. La unidad didáctica propuesta pretende desarrollar no solo la comprensión de los contenidos científicos sino también cómo se construyen por medio de las prácticas científicas, por lo que constituye una alternativa innovadora para el aprendizaje de estos contenidos con sentido para el alumnado (Couso, 2013).

Según los comentarios del alumnado, los resultados de la prueba piloto fueron bastante prometedores y, además, permitieron introducir ciertas mejoras. Inicialmente, la propuesta era más amplia, por lo que se consideró oportuno estructurarla en sesiones de menor duración, así como modificar el orden de las actividades con la finalidad de que la secuenciación fuera más adecuada. Además, ha sido necesario modificar algunos de los diseños, como el indicador de $\mathrm{pH}$ empleado en la actividad 3 para alcanzar un cambio más visual.

Resulta pertinente, sin embargo, destacar la necesidad de evaluar la propuesta mediante su implementación en el aula con un mayor número de estudiantes de 2 ㅇ y 3 o curso de Educación Secundaria para evidenciar el potencial real de la misma.

\section{Agradecimientos}

Los autores agradecen el proyecto PGC2018-097988-A-I00 financiado por: FEDER / Ministerio de Ciencia e Innovación (MCI) de España-Agencia Estatal de Investigación (AEI).

\section{Referencias bibliográficas}

Andersson, B. (1990). Pupils' conceptions of matter and its transformations (age 12-16). Studies in Science Education, 18, 53-85. DOI: https://doi. org/10.1080/03057269008559981

Aragón, M.M., Oliva-Martínez, J.M. y Navarrete, A. (2013). Evolución de los modelos explicativos de los alumnos en torno al cambio químico a través de una propuesta didáctica con analogías. Enseñanza de las Ciencias, 31(2), 9-30. DOI: https://doi. org/10.5565/rev/ec/v31n2.832

Bayram-Jacobs D, Henze I, Evagorou M, Shwartz, Y., Aschim, E. L., Alcaraz-Dominguez, S., Barajas, M. y Dagan, E. (2019). Science teachers' pedagogical content knowledge development during enactment of socioscientific curriculum materials. Journal of Research in Science Teaching, 1-27. DOI: https://doi.org/10.1002/tea.21550

Bellová, R., Melichercíková, D. y Tomcík, P. (2018). Possible Reasons for Low Scientific Literacy of Slovak Students in Some Natural Science Subjects. Research in Science \& Technological Education, 36(2), 226-242

Blanco, A., España, E. y Rodríguez, F. (2012). Contexto y enseñanza de la competencia científica. Alambique, 70, 9-18.

Boesdorfer, S. B. y Livermore, R. A. (2018). Secondary school chemistry teacher's current use of laboratory activities and the impact of expense on their laboratory choices. Chem. Educ. Res. Pract., 19, 135-148. 
Boghian, I., Cojocariu, V.-M., Popescu, C. V. y Mâtă, L. (2019). Game-based learning. Using board games in adult education. Journal of Educational Sciences \& Psychology, IX (LXXI), 51-57.

Caamaño, A. y Oñorbe, A. (2004). La enseñanza de la química: conceptos y teorías, dificultades de aprendizaje y replanteamientos curriculares. Alambique, 41, 68-81.

Caamaño, A. (2018). Enseñar química en contexto: un recorrido por los proyectos de química en contexto desde la década de los 80 hasta la actualidad. Educación Química, 29, 21-54.

Couso, D. (2013). La elaboración de unidades didácticas competenciales. Alambique, 74, 12-24.

Crujeiras, B, Jiménez-Aleixandre, M. P. y Gallástegui, J. R. (2013) Indagación en el laboratorio de química. Secuencia de actividades en que el alumnado de 3 ㅇ y 4 으 de ESO diseñan experimentos. Alambique, 74, 49-56

Crujeiras Pérez, B. y Jiménez-Aleixandre, M. P. (2018). Influencia de distintas estrategias de andamiaje para promover la participación del alumnado de secundaria en las prácticas científicas. Enseñanza de las ciencias, 36(2), 23-42. DOI: https://doi. org/10.5565/rev/ensciencias.2241

Domínguez, C., Azcona, R. y Guisasola, J. (2000). La enseñanza y el aprendizaje del conocimiento químico. En F.J. Perales y P. Cañal (Dir.), Didáctica de las Ciencias Experimentales. Teoría y Práctica de la Enseñanza de las Ciencias. Alcoy: Editorial Marfil, 421-450.

Domènech-Casal, J. (2017). Propuesta de un marco para la secuenciación didáctica de Controversias Socio-Científicas. Estudio con dos actividades alrededor de la genética. Revista Eureka sobre Enseñanza y Divulgación de las Ciencias, 14(3), 601-620. DOI: http://dx.doi.org/10.25267/Rev_Eureka_ensen_divulg_cienc.2017.v14.i3.07

Franco-Mariscal, A.J., Blanco-López, A. y España-Ramos, E. (2014). El desarrollo de la competencia científica en una unidad didáctica sobre la salud bucodental. Diseño y análisis de tareas. Enseñanza de las Ciencias, 32(3), 649-667. DOI: https://doi. org $/ 10.5565 / \mathrm{rev} / \mathrm{ensciencias.1346}$

Franco, A. J y Oliva, J. M. (2013), Diseño de una unidad didáctica sobre los elementos químicos, Alambique, 74, 57-67.

Furió, C. y Furió, C. (2000). Dificultades conceptuales y epistemológicas en el aprendizaje de los procesos químicos. Educación Química, 11(3), 300-308. DOI: http://dx.doi. org/10.22201/fq.18708404e.2000.3.66442

Garrido Espeja, A. y Couso Lagarón, D. (2013). La competència d'ús de proves científiques: Quines dimensions es promouen a les activitats de l'aula de ciències? Ciències, 24, 42-47.

Gee,J.P. (2003). What Video Games Have to Teach UsA boutLearning and Literacy. Technology Pedagogy and Education, 1(1), 20. DOI: https://doi.org/10.1145/950566.950595

Ibacache Plaza, M. y Merino Rubilar, C. (2021). Una propuesta de secuencia basada en el contexto, para la promoción de la argumentación científica en el aprendizaje de las reacciones químicas con estudiantes de educación media técnico profesional. Revista Eureka sobre Enseñanza y Divulgación de las Ciencias, 18(1), 1105. DOI: https://doi.org/10.25267/Rev_Eureka_ensen_divulg_cienc.2021.v18.i1.1105 
Jiménez-Aleixandre, M. P. (2010). 10 ideas clave. Competencias en argumentación y uso de pruebas. Barcelona: GRAO

Jiménez-Liso, M. R. (2020). Aprender ciencia escolar implica aprender a buscar pruebas para construir conocimiento (indagación). En D. Couso, M.R. Jimenez-Liso, C. Refojo y J.A. Sacristán (Coords), Enseñando Ciencia con Ciencia. FECYT \& Fundacion Lilly. Madrid: Penguin Random House.

Jiménez-Liso, M. R., Martínez-Chico, M. y Salmerón-Sánchez, E. (2018). Chewing Gum and $\mathrm{pH}$ Level of the Mouth: A Model-based Inquiry Sequence to Promote Scientific Practices. World Journal of Chemical Education, 6(3). DOI: https://doi.org/10.12691/ wjce-6-3-2

Jong, O. De (2008) Context-based chemical education: how to improve it? Chemical Education International, 8, 1-7.

Jorba, J. y Sanmartí, N. (1994). Un dispositivo pedagógico que incorpora la regulación continua de los aprendizajes. En J. Jorba y N. Sanmartí (Eds.), Enseñar, aprender y evaluar: un proceso de evaluación continua, 33-94. Barcelona: Ministerio de Educación y Cultura.

Marchán-Carvajal, I. y Sanmartí, N. (2015). Criterios para el diseño de unidades didácticas contextualizadas: aplicación al aprendizaje de un modelo teórico para la estructura atómica. Educación Química, 26, 267-274.

Mehta, G., Yam, V. W. W., Krief, A, Hopf, H. y Matlin, S. A. (2018). The Chemical Sciences and Equality, Diversity, and Inclusion. Angewandte Chemie-International Edition, 57(45), 14690-14698. DOI: https://doi.org/10.1002/anie.201802038

Ministerio de Educación, Cultura y Deporte (2015). Real Decreto 1105/2014, de 26 de diciembre, por el que se establece el currículo básico de la Educación Secundaria Obligatoria y del Bachillerato.

Pujol, R. M. (2003). Didáctica de las ciencias en la educación primaria. Madrid: Síntesis Educación.

Sanmartí, N. (2000). El diseño de unidades didácticas. En F.J. Perales y P. Cañal (Eds), Didáctica de las ciencias experimentales, , 239-266.

Strippel, C.G. y Sommer, K. (2015). Teaching Nature of Scientific Inquiry in Chemistry: How do German chemistry teachers use labwork to teach NOSI? International Journal of Science Education, 37(18), 2965-2986. DOI: https://doi.org/10.1080/09500693.201 5.1119330

Talanquer, V. (2006). Commonsense Chemistry: A Model for Understanding Students' Alternative Conceptions. Journal of Chemical Education, 83(5), 811. DOI: https:// doi.org/10.1021/ed083p811

Talanquer, V. y Pollard, J. (2010). Let's teach how we think instead of what we know. Chemical Education Research and Practice, 11, 74-83. DOI: https://doi.org/10.1039/ C005349J

Talanquer, V. (2013). School Chemistry: The Need for Transgression. Science \& Education, 22, 1757-1773. DOI: https://doi.org/10.1007/s11191-011-9392-x

Zhang, D. y Campbell, T. (2012). An Exploration of the Potential Impact of the Integrated Experiential Learning Curriculum in Beijing, China. International Journal of Science Education, 34(7), 1093-1123. DOI: https://doi.org/10.1080/09500693.2011.625057 


\section{Material anexo.}

\section{Descripción de las actividades de la unidad didáctica}

Actividad 1. ¿Es cierto que España se convertirá en un gran desierto? Científicos/as que investigan sobre el efecto invernadero y sus consecuencias relacionadas con el cambio climático

Esta primera actividad tiene como objetivo contextualizar la unidad didáctica en un tema de actualidad e interés para el alumnado, así como de familiarizarlos con las científicas y científicos que han investigado sobre los contenidos relacionados con el efecto invernadero y sus consecuencias, destacando cuáles son sus valores y sus aportaciones, y favoreciendo una actitud positiva frente a la ciencia al tiempo que se da visibilidad al papel de la mujer en este campo.

El recurso principal se trata de una presentación en la plataforma virtual Genially (figura 2) en la que se incorporan enlaces interactivos a los que accederán de forma individual. En cada uno de los enlaces dispondrán del perfil de una científica/o y del trabajo que ha desarrollado. A continuación, representarán mediante un juego de rol a dicho/a investigador/a, haciendo referencia a aspectos relacionados con la sociedad de la época, dificultades que se les presentaban y controversias relacionadas con su investigación.

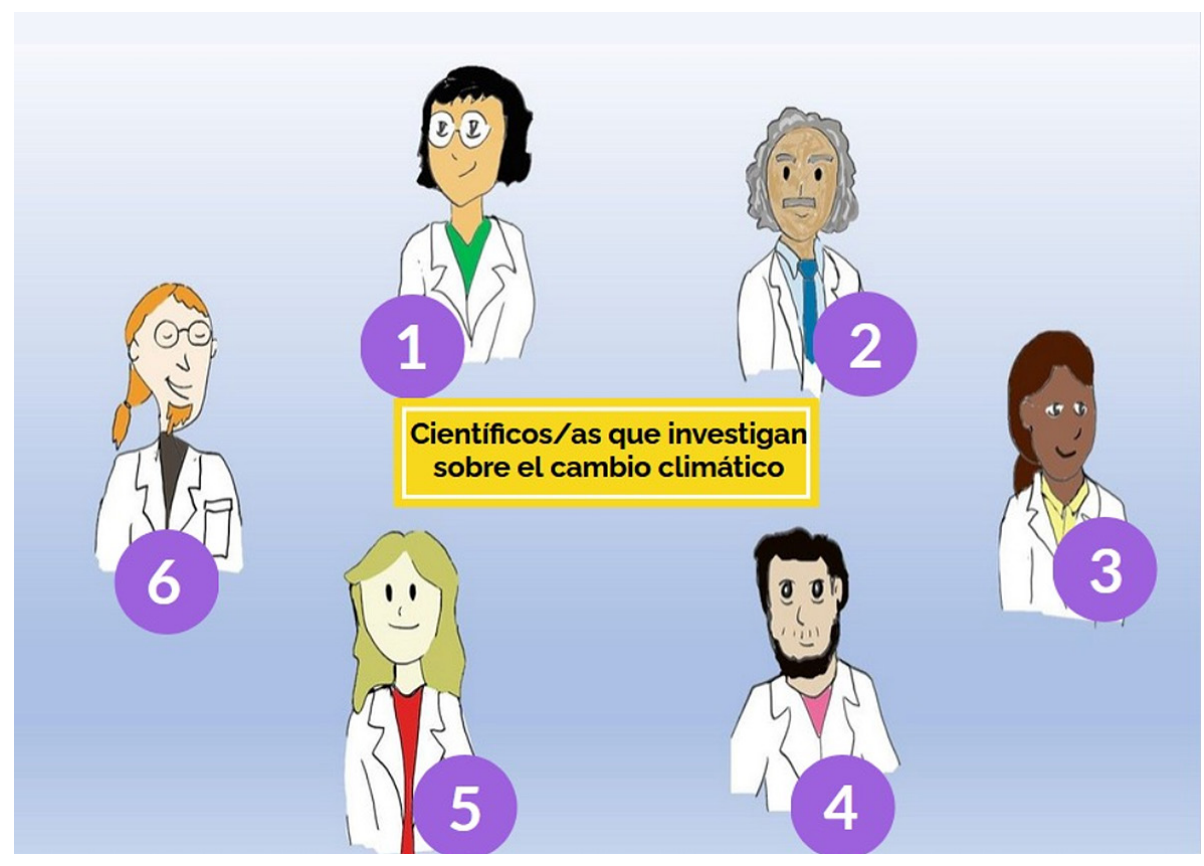

Figura 2. Investigadores/as del efecto invernadero

https://view.genial.ly/5e568c5d9f29960f080fce60/interactive-image-cientificos-cambio-climatico

Los científicos y científicas incluidos en esta propuesta por la relevancia de su trabajo en dicho campo son: Eunice Foote, Svante Arrhenius, Pilar Dorado, Gene Likens, Leen Peeters y David Edward Archer. 
Actividad 2. ¿Cómo podríamos averiguar si los gases que expulsan los vehículos hacen que aumente la temperatura por efecto invernadero?

Enlazamos la actividad anterior con una propuesta para destacar el papel de Eunice Foote, científica que en 1856 teorizaba por vez primera sobre la existencia del efecto invernadero y que iba a presentar en la octava reunión anual de la Asociación Estadounidense para el Avance de la Ciencia (AAAS).

En la misma, se anima al alumnado a que realice un diseño experimental que les permita comprobar el efecto del $\mathrm{CO} 2$ en la temperatura, guiados por el profesorado, por medio de un enfoque de enseñanza por indagación y modelización.

Entre los materiales, se hace uso de una lámpara de radiación infrarroja y sensores de temperatura. El alumnado puede hacer las medidas experimentales que considere y observar cómo va cambiando la temperatura con el tiempo, registrando los valores automáticamente en una gráfica (figura 3). Durante la actividad tendrán que ir completando el cuaderno de trabajo que les servirá de guía durante la investigación.

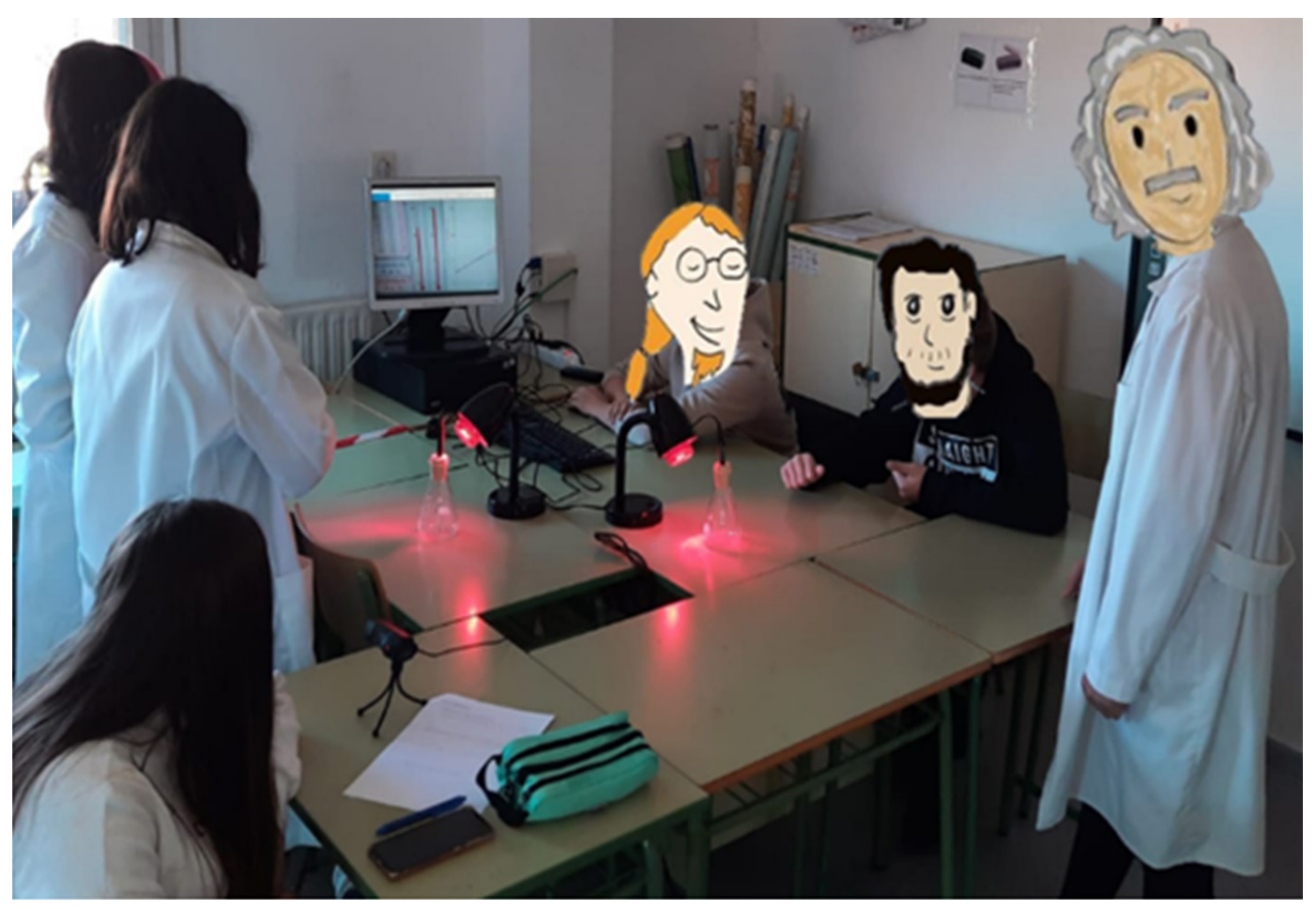

Figura 3. Fotografía del montaje realizado. 
Actividad 3. Generación de la lluvia ácida.

La siguiente actividad de la fase de desarrollo está relacionada con la producción de $\mathrm{CO} 2$ mediante una reacción de combustión de la madera, así como SO2 por la de una cabeza de cerilla. Con esta actividad se pretende acercar al alumnado el concepto de lluvia ácida mediante la realización de una experiencia que simula cómo se produce en la atmósfera y qué efectos tiene sobre el entorno (figura 4).
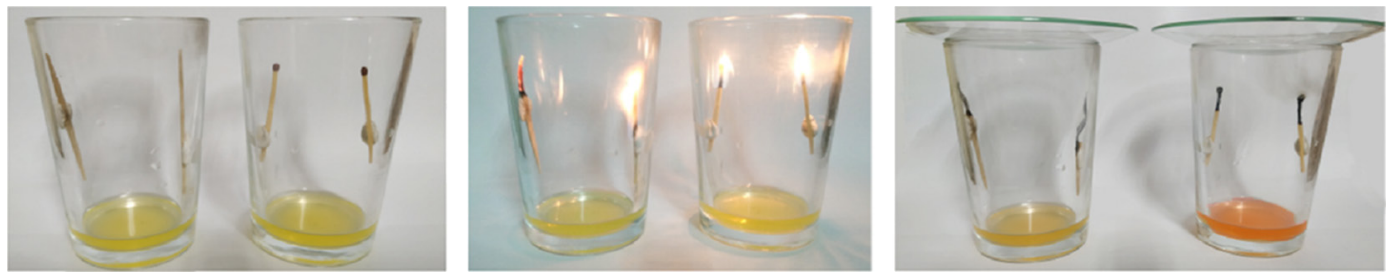

Figura 4. Combustión de madera y cerilla, respectivamente.

Además, se relacionará con el concepto de $\mathrm{pH}$, el cual será medido cualitativamente mediante 10 $\mathrm{ml}$ de una disolución del indicador naranja de metilo que virará entre amarillo/anaranjado ( $\mathrm{pH} 4.4)$ y un color rojizo ( $\mathrm{pH}$ 3.1). En la pared de los vasos que contienen estas disoluciones, y con ayuda de un material similar a la plastilina, se adhieren dos astillas de madera o dos cerillas, las cuales se prenden para que se inicie la combustión y se tapan con sendos vidrios de reloj. Al quemar las cerillas, el azufre que contienen es oxidado dando lugar a dióxido de azufre (SO2). Cuando el gas se disuelve en el agua, forma ácido sulfúrico (H2SO4) que reduce el pH de la disolución y provoca el cambio de color del indicador.

$$
\mathrm{SO} 2(\mathrm{~g})+1 / 2 \mathrm{O} 2(\mathrm{~g})+\mathrm{H} 2 \mathrm{O}(\mathrm{I}) \rightarrow \mathrm{H} 2 \mathrm{SO} 4(\mathrm{I})
$$

En el caso de las astillas, el gas liberado es $\mathrm{CO} 2$. Su solubilización en agua genera $\mathrm{H} 2 \mathrm{CO} 3$, que también tiene carácter ácido, pero en menor medida, por lo que el viraje que sufre el indicador es más tenue. La fortaleza de ambos ácidos, y por tanto su grado de disociación, se comprueba al final de la experiencia midiendo el $\mathrm{pH}$ de las disoluciones resultantes. Los cambios observados serán registrados en un cuaderno de laboratorio de carácter formativo en el que el alumnado debe identificar el tipo de reacción química que se produce en la experiencia, dibujar el diseño experimental, recoger e interpretar los datos, describir el fenómeno observado, incluir las posibles explicaciones, realizar una representación simbólica y extraer las conclusiones (figura 5).

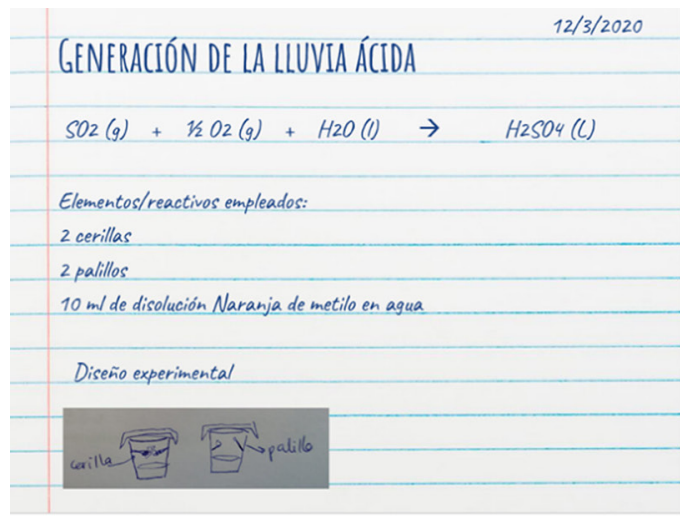

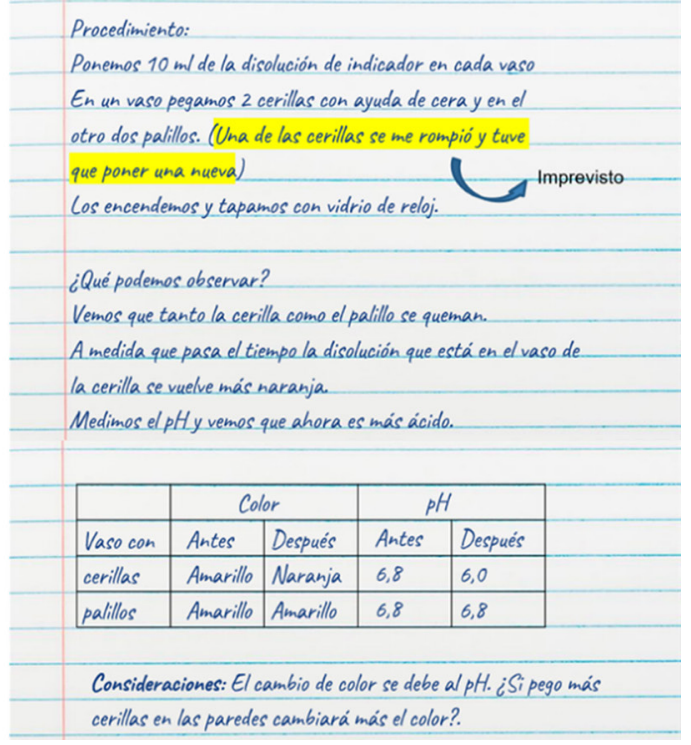

Figura 5. Ejemplo de cuaderno de laboratorio. 
Actividad 4. ¿Duran menos los monumentos por causa de la lluvia ácida?

Como actividad de la fase de aplicación emplearemos una segunda actividad con enfoque de enseñanza por indagación. En este caso plantearemos la pregunta que se ha seleccionado para el título de la actividad con la intención de comprobar si el alumnado es capaz de desarrollar una investigación sobre las consecuencias que tiene el contacto de la lluvia ácida sobre los edificios y monumentos de forma autónoma. Al encontrarnos en la fase de aplicación, la intencionalidad es evaluar la competencia científica en relación a la formulación de hipótesis, planificación de un diseño experimental, análisis y recogida de datos y establecimiento de conclusiones. Es de gran relevancia que sean capaces de observar algún cambio característico que indique que está teniendo lugar una reacción química (como es la disolución de un sólido y formación de una sustancia gaseosa). Los materiales de los que dispondrán son tiza, vasos, ácido acético, agua, limón, papel indicador de pH (figura 6).

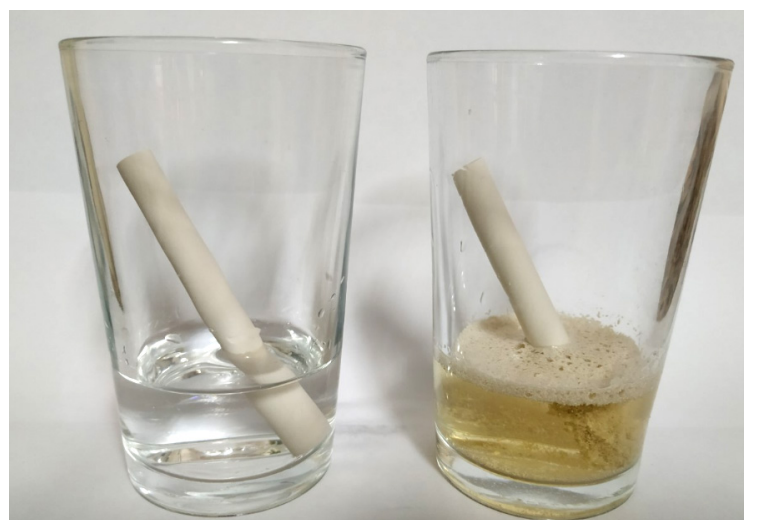

Figura 6. combustión de madera y cerilla, respectivamente.

Esta actividad tendrá una doble finalidad evaluativa: i) de formación, al favorecer la reflexión del alumnado y regular su aprendizaje; y ii) de calificación, al ser de finalización de la unidad y disponer de un cuaderno de laboratorio mediante el que se podrán evaluar los conocimientos imprescindibles para realizar investigaciones científicas. Esta evaluación permitirá, además, regular el proceso de enseñanza y recoger propuestas de mejora dentro del proceso de enseñanza-aprendizaje. 
Actividad 5. Chem's up

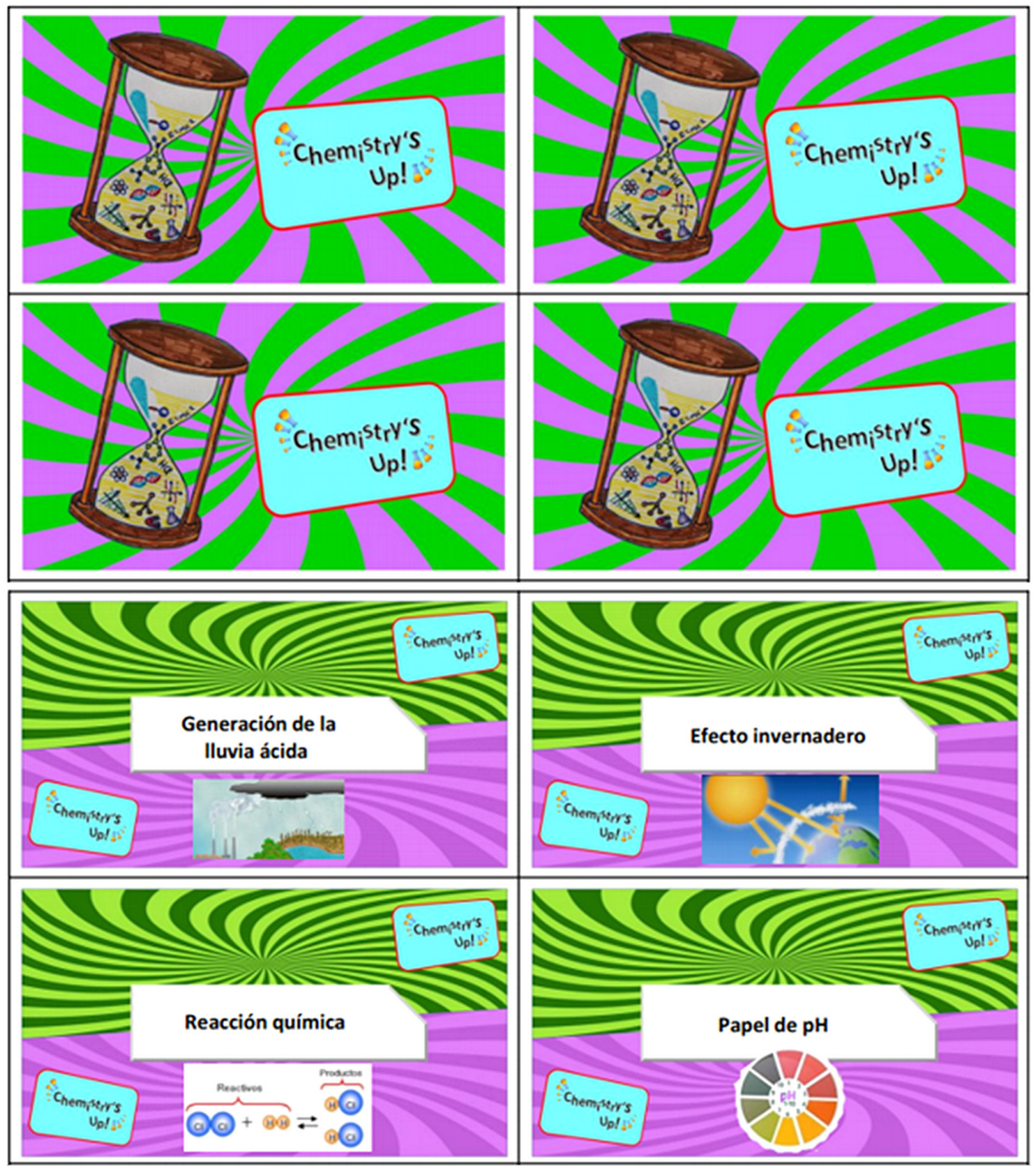

Figura 7. Ejemplo de algunas de las cartas del juego Chem's up.

Para la fase de revisión se diseña el juego de cartas Chem's up, basado en el popular Time's up. Se pretende que con esta actividad puedan revisar, de forma lúdica y adecuada para su nivel educativo (2--3을 ESO), los contenidos trabajados en la unidad didáctica. Algunos ejemplos de contenidos a revisar son: reacción química, efecto invernadero, lluvia ácida, enrasar una probeta, diseño experimental, formulación de hipótesis, etc. (Figura 7)

El juego consiste en dividir al alumnado en dos equipos, los cuales deben acertar el mayor número de tarjetas posibles en el tiempo del que disponen. Se desarrolla en cuatro rondas y, aunque en todas ellas se emplean las mismas tarjetas, varía la forma en la que representan los contenidos, comenzando con la elección de cualquier palabra, excepto aquellas que tengan la misma raíz que el contenido que se pretende definir. A continuación, en la segunda ronda se limitará a una sola palabra, en la tercera ronda se recurre a la mímica y, en la última ronda, se debe realizar un único movimiento capaz de representar el contenido de la tarjeta. El equipo que acumule más tarjetas ganará la ronda.

Al final de las cuatro rondas, el equipo que acierta más tarjetas será el ganador. La propia actividad servirá como evaluación sumativa tanto al profesorado como al alumnado, al ser conscientes de los conocimientos que han adquirido y aprender interactuando colectivamente. 
Actividad 6. ¿La actividad humana es la causante del ascenso de la temperatura del planeta? ¿Tienen razón los negacionistas?

En esta actividad se propone introducir una noticia de actualidad (https://elpais.com/ elpais/2019/09/18/eps/1568820907_023534.html) acerca de las opiniones de personas de diferentes ámbitos acerca del ascenso de la temperatura del planeta para argumentar, utilizando los contenidos adquiridos en las actividades de desarrollo, sobre los efectos de los gases en el ascenso de la temperatura del planeta, relacionándolo con el concepto de cambio climático. En concreto, para fomentar la argumentación y evaluar cómo el alumnado justifica diferentes posturas, se propone leer documentación complementaria a la noticia de prensa y utilizar las ideas científicas adquiridas en la unidad didáctica para contestar, en pequeños grupos, a las siguientes cuestiones:

1- ¿Qué ventajas tiene utilizar las reservas de hidrocarburos para la producción de energía y otros productos de uso cotidiano?

2- ¿Qué inconvenientes (médico, comercial, ambiental...) se atribuyen a la utilización de los hidrocarburos?

3- ¿Considerarías necesario que en el embalaje de los productos cotidianos se indique la procedencia y gasto energético en su producción?

4- ¿Conoces cuál es tu consumo de luz y otras fuentes energéticas? ¿Te gustaría conocerlo?

A continuación, se realizará una puesta en común para fomentar el debate colectivo. Finalmente, de forma individual, se deben redactar un ensayo, expresando la opinión personal al respecto, que contenga argumentos que apoyen y refuten la teoría. Para ello, es preciso partir de las ideas científicas que se han comentado en la unidad didáctica, así como en aspectos de valor (de tipo ético, moral, salud, ambiental). 